\title{
Ponthieva nicolasii (Orchidaceae), una nueva especie para Hidalgo, México
}

\section{Ponthieva nicolasii (Orchidaceae), a new specie from Hidalgo, Mexico}

\author{
Vincenzo Bertolini ${ }^{1 *}$ \& Fredy Archila Morales ${ }^{2,3}$ \\ ${ }^{1}$ El Colegio de la Frontera Sur, unidad Tapachula, carretera antiguo aeropuerto km 2.5, Colonia Centro, Tapachula, Chiapas, \\ México. \\ ²Estación Experimental de Orquídeas de Guatemala, 1 Av. 5-28 zona 1. Cobán A.V. Guatemala C.A. \\ ${ }^{3}$ Herbario BIGU, Universidad de San Carlos de Guatemala, Campus central edificio T-10, segundo nivel, Escuela de Biología, \\ Facultad de Ciencias Químicas y Farmacia, zona 12, Guatemala. \\ *vin.bertolini@gmail.com
}

\begin{abstract}
Field studies in the Mezquital Valley of the state of Hidalgo, Mexico, allowed to locate a population of a specie belonging to the genus Ponthieva R.Br. Comparing the specifies entity to other species of Mexican Ponthieva, we conclude that it is a new taxon, with strong differences between it and the other ones. We report taxonomic diagnosis, descriptor and botanical icon for acceptance.
\end{abstract}

La familia Orchidaceae es uno de los grupos taxonómicos más diverso y amplio en número de especies, estimadas entre 25.000 y 35.000 (Dressler 1990). México es un país megadiverso, por su variedad fisiográfica y climática (Rzedowski 2006). Se estima que la orquideoflora mexicana comprende más de 1.200 especies y subespecies, distribuidas en 164 géneros (Soto-Arenas et al. 2007). Una de sus características más sobresalientes es la proporción de endemismos, con 444 especies o subespecies endémicas, aproximadamente el $40 \%$ del total de taxa (Soto-Arenas 1996). A nivel nacional, 181 especies se encuentran en alguna categoría de riesgo, de los cuales 74 se consideran amenazadas o en peligro de extinción (SEMARNAT 2010). El estado de Hidalgo alberga un total de 73 taxa de Orchidaceae reportados; en el Valle del Mezquital se han encontrado 20 de ellos, de los cuales solamente 4 tienen hábito vegetativo epífito (Bertolini et al. 2012).

El estado de Hidalgo se ubica en la parte más sur de la ecozona Neartica, teniendo áreas de transición de trópico árido y subtrópico (Schultz 2002). El clima es semiárido con una precipitación promedio alrededor de $400 \mathrm{~mm}$ por año (INEGI 2008). No obstante, a pesar que su extensión representa el $1 \%$ del territorio nacional $\left(20.905,12 \mathrm{~km}^{2}\right)$, posee una diversidad ecológica amplia, gracias a su variabilidad en topografía y condiciones microclimaticas, hospedando el $10 \%$ de la flora mexicana (Pérez-Escandón et al. 2003). El Valle del Mezquital presenta un tipo de vegetación de matorral xerofítico ricos en cactáceas y bosque espinoso de mezquites (Prosopis laevigata (Willd.) M.C.Johnst.) y huizaches (Acacia farnesiana (L.)
Willd.). Este tipo de vegetación varía gradualmente al bosque pino-encino, conforme se suba de latitud y altitud en el Alto Mezquital hasta llegar a la Sierra Gorda (Rzedowski 2006). El sitio del hallazgo, geográficamente se coloca en el Alto Mezquital/Sierra Gorda, en la ecorregión de la Sierra Madre Oriental con bosque pino-encino, según las "Ecorregiones Terrestres Mundiales" del WWF (Olson et al. 2001) (Fig. 1).

Uno de los géneros de la subtribu Cranichidinae Lindl. es Ponthieva R.Br., posee 62 especies (WCSP 2015), simpodiales, terrestres o litófitas, distribuidas desde elevaciones bajas y altas, colinas, bosques lluviosos montanos, bosques con manchones de Sphagnum y matorrales, desde el sureste de los Estados Unidos hasta el noroeste de Argentina (Alrich \& Higgins 2008). Ponthieva posee un ginostemio corto, erecto, relativamente masivo, a menudo hinchado en el ápice; la columna es más corta que la antera, pie ausente; base de la antera situada debajo de la base del estigma, antera erecta, oblonga u ovada, móvil, biseptadas, septos paralelos; filamento entero o en la mayor parte fusionado con la parte de la columna; conectivo estrecho pero muy grueso a menudo apicalmente engrosado; polinias en número de 4, ovoideo a oblongoovoideo, compactas; caudículas inconspicuas, formadas de ápices de polinias delgadas; estaminodios unidos al filamento y el estilo y/o la base del estigma, formando un clinandrio dorsal prominente, usualmente grueso, masivo, espacioso; estigma horizontal a subventral, obscuramente 3-lobado, confluente, oval o elíptico (Szlachetko \& Rutkowski 2000, Archila et al. 2015). 


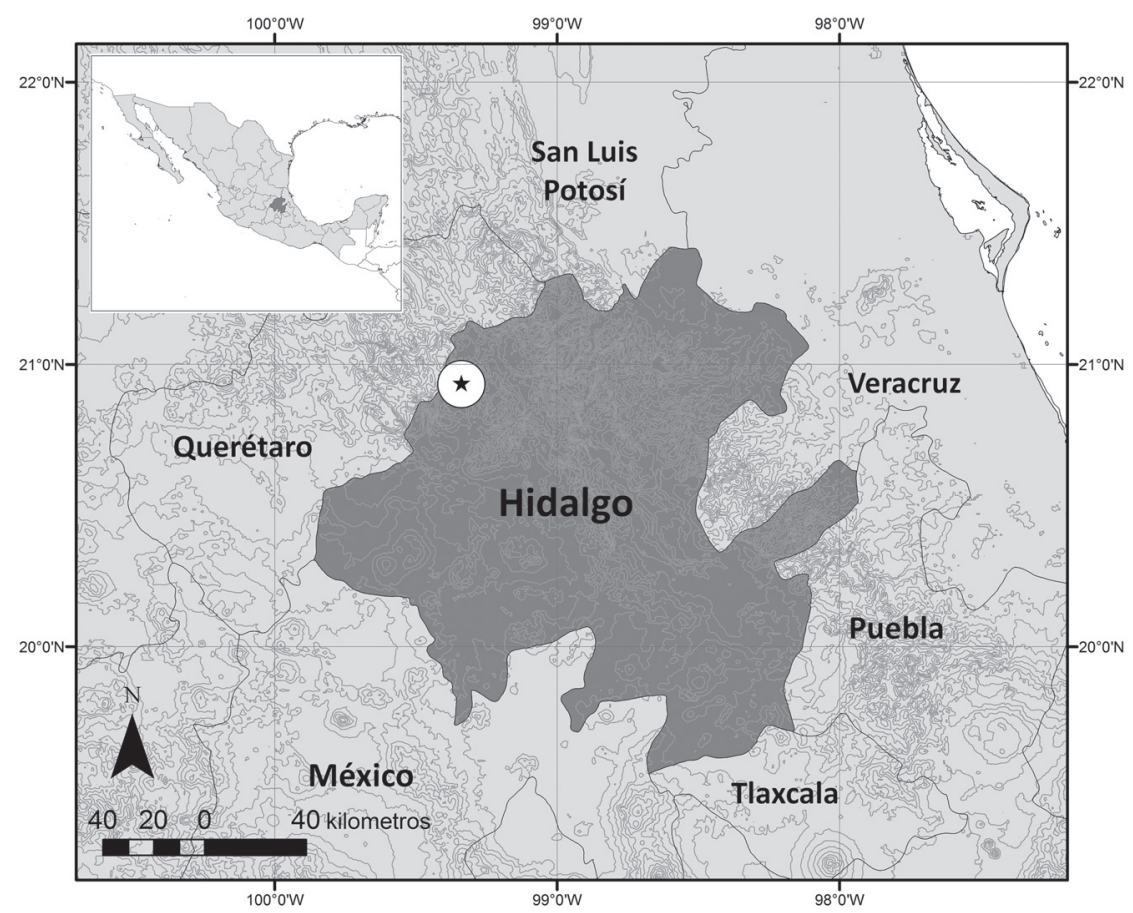

Figura 1. Sitio de estudio, Estado de Hidalgo, México. Sitio de recolección indicado en un círculo con estrella.

FIGURE 1. Site location, State of Hidalgo Mexico. The star-circle is the point of plants recollection.

La especie reportada se encontró por la primera vez en el año 2009, durante salidas de campo por un inventario botánico por parte de la Universidad Tecnológica del Valle del Mezquital, y se mantuvo bajo observación anual hasta el 2015. Analizando el material recolectado se determinó que se trata de una nueva especie, por poseer patrones taxonómicos distintivos.

Ponthieva nicolasii Archila \& Bertolini, sp. nov. TIPO: México, estado de Hidalgo, municipio de Pacúla, poblado de Milpas Viejas, 20 56 '20,41" N - 99 20'53,05" O, 1889 m s.n.m., V. Bertolini n 1, 15-XI-2013 (Holotipo HGOM).

Herba terrestris nana. Flos albo-viridea. Haec herba P. shaffneri similis est sed inflorescentia bracteis floribusque leviter pubescentis, floribus minoribus. Fructis pubescentis, petalis dolabriformis (versus linearibus), apice rotundis (versus acutis). Petalorum limborum lobo rotundum. Sepalis lateralibus orbicularis divergentibus.

Hierba terrestre, de hasta $30 \mathrm{~cm}$ de altura en plena floración. Raíces de 3 a 5, con un diámetro de $2-4 \mathrm{~mm}$, suculentas, color blanco/beige, con escasos pelos radicales localizados sobre todo en el primer centímetro a partir del ápice. Hojas de 3 a 5 , visibles al momento de la floración, de $4,5 \mathrm{~cm}$ de largo por 2,6 cm de ancho. Inflorescencia de 15 a $25 \mathrm{~cm}$ de largo, diámetro del vástago de $1,5-1,8 \mathrm{~mm}$ a los $10 \mathrm{~cm}$ de la roseta. Pedúnculo de la inflorescencia, con los botones florales cerrados, de 1,5 $\mathrm{mm}$ de largo. Flores no resupinadas, con pubescencia capitada en la parte externa de los sépalos y el ápice de los pétalos. Sépalo superior oblicuamente linear-elíptico, con el ápice acuminado, de 7,5 $\mathrm{mm}$ de largo por $3 \mathrm{~mm}$ de ancho. Sépalos laterales orbiculares agudos, de 5-5,2 $\mathrm{mm}$ de largo por 2,5-3,5 $\mathrm{mm}$ de ancho. Pétalos subdolabriformes, algo espatulados, con el ápice redondeado, de 4,8-5 $\mathrm{mm}$ de largo por $0,9 \mathrm{~mm}$ de ancho. Ovario geniculado pedicelado, elíptico, globoso, pubescente, de $11 \mathrm{~mm}$ de largo por $0,7 \mathrm{~mm}$ de ancho. Bráctea floral, oblonga con el ápice acuminado, de 5,8 $\mathrm{mm}$ de largo y 2,8 $\mathrm{mm}$ de ancho. Gynostemio oblicuamente redondeado, de $2 \mathrm{~mm}$ de largo y $1 \mathrm{~mm}$ de ancho. Labelo pedicelado, trilobado, los lobos laterales pequeños redondeados, el lobo central oblongo con el ápice oblongo, agudo, engrosado. Labelo de 2,4-2,7 $\mathrm{mm}$ de largo por $1 \mathrm{~mm}$ de ancho máximo, manteniendo su forma natural (2,2 con los lóbulos, aletas, del labelo abierto manualmente), a la base de $0,1 \mathrm{~mm}$ de ancho (Figs. 2 y 3 ).

Etimología: El epíteto específico se dedica a Nicolás Bertolini Luna, hijo de uno de los autores.

P. nicolasii es una especie del Alto Mezquital de Hidalgo, México, muy afín morfológicamente con Ponthieva schaffneri (Rchb. F.) E.W. Greenw., de la que se diferencia porque su ovario es geniculado, los pétalos son dolabriformes, el sépalo superior es más pequeño y 
elíptico, los sépalos laterales son divergentes. El sitio de recolección está caracterizado por un bosque degradado de enebro-encino, con mediana-alta presión antrópica por actividad ganadera. Los elementos arbóreos son de gran tamaño, con un diámetro de aprox. 1,5 $\mathrm{m}$ y altura alrededor de $15 \mathrm{~m}$. La topografía del sitio y su altitud propician la acumulación de precipitaciones ocultas (roció nocturno y matutino), formando microcuencas frescas y húmedas. En estos rincones se refugia $P$. nicolasii, asociada a grandes cantidades de musgos y Sellaginella sp., además de plantas carnívoras de Pinguicula moranensis Kunth.
La población ha tenido un número de individuos que ha fluctuado de 5 a 10 durante el periodo de observación experimental, y distribuida en el sitio de forma dispersa. $\mathrm{Su}$ periodo de floración ha sido relativamente amplio, presentándose desde octubre a diciembre. En el sitio del hallazgo se ha podido observar la presencia de Ponthieva schaffneri, al contrario nunca se ha registrado P. racemosa, durante el periodo de observación.

La nueva especie se diferencia de las otras dos registradas para el estado de Hidalgo (Bertolini et al. 2012), Ponthieva racemosa (Walter) C. Mohr y P. schaffneri (Rchb.f.) E.W. Greenw., con la siguiente clave dicotómica:

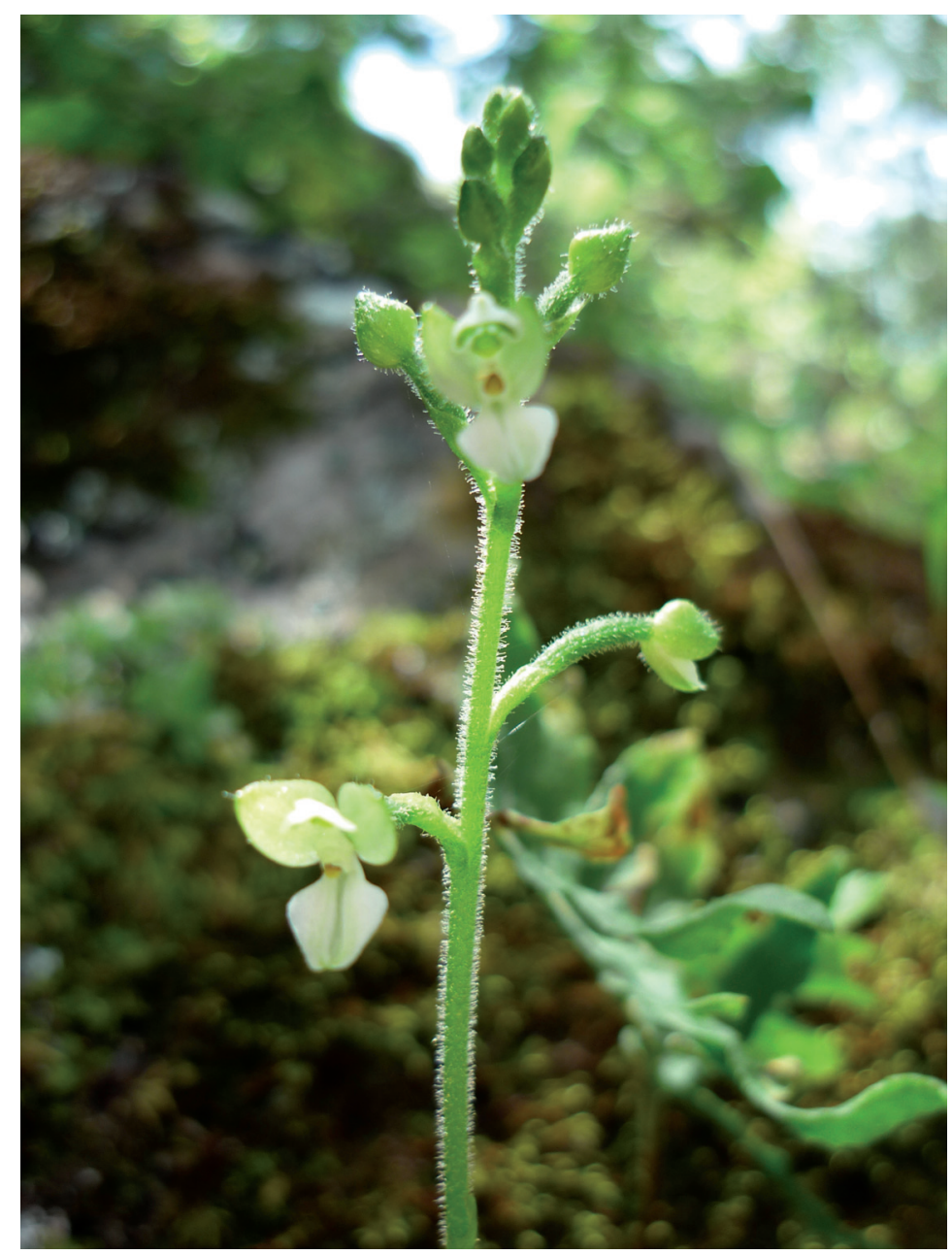

Figura 2. Plena floración, flores totalmente abiertas.

FIGURE 2. Full blooming, flowers totally opened.

1. Ginostemio con la base de la columna oblicuamente cuadrada. 


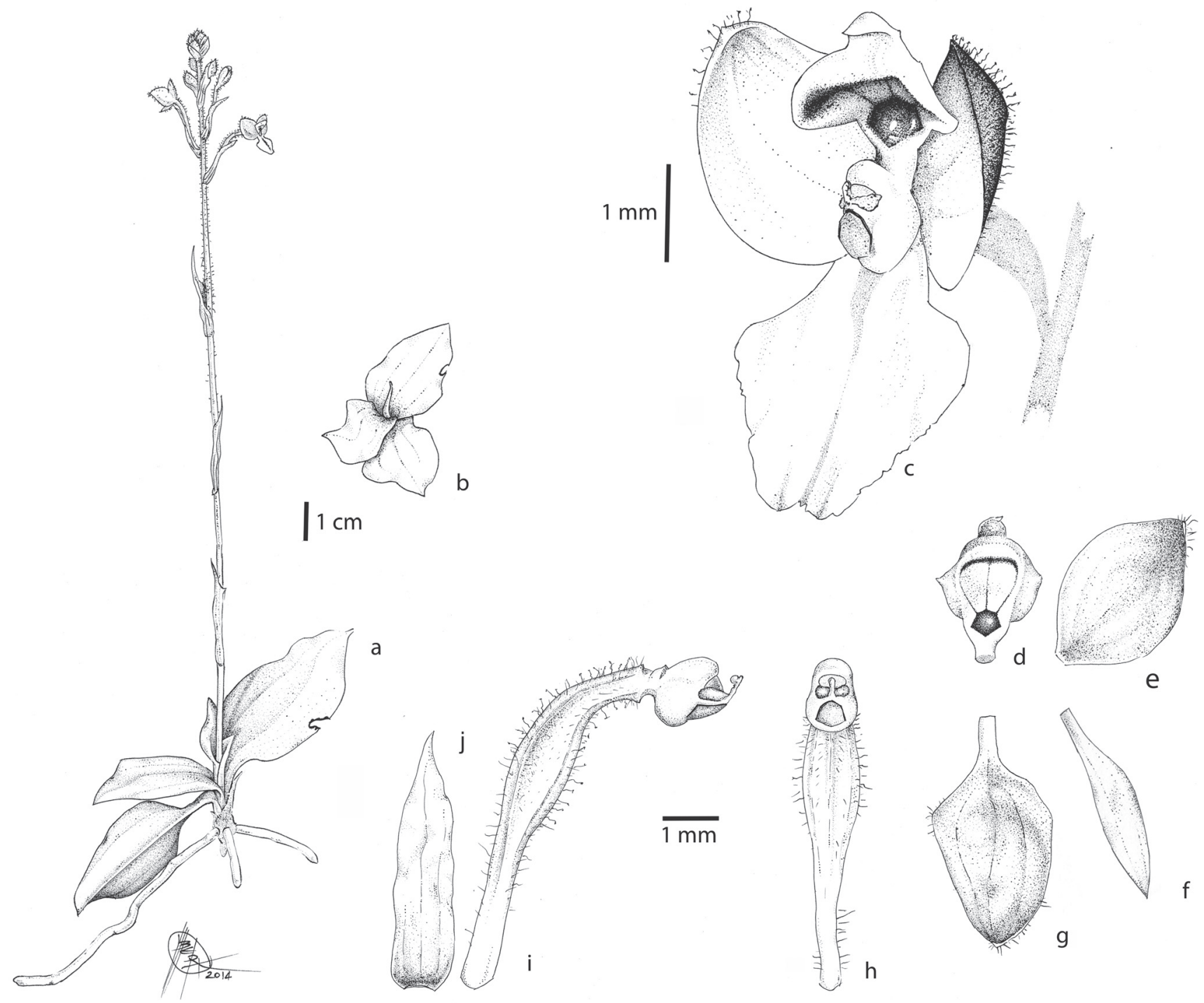

Figura 3. Ponthieva nicolasii Archila \& Bertolini, ícono botánico: a) planta adulta en plena floración; b) roseta vegetativa juvenil; c) flor completa; d) labelo; e) sépalo; f) pétalo; g) sépalo dorsal; h) columna y ovario, vista frontal; i) columna y ovario, vista lateral; j) bráctea floral.

Figure 3. Ponthieva nicolasii Archila \& Bertolini, botanical drawing: a) adult plant in full blooming; b) rosetta of young plant; c) flower; d) label; e) sepal; f) petal g) dorsal sepal; h) column and ovary, front view; i) column and ovary, side view; j) floral bract.

\section{AGRADECIMIENTOS}

Sumamente agradecidos al historiador y amigo Francisco Ramiro Luna Tavera ( $\dagger$ ), que en paz descanse, y a los habitantes de las comunidades que nos han brindado apoyo logístico durante los recorridos.

\section{BIBLIOGRAFÍA}

Alrich, P. \& W. Higgins. 2008. Illustrated Dictionary of Orchid Genera. Selby Botanical Gardens Press, Sarasota FL. 482 pp.

Archila, F., D. Szlachetko, G. Chiron \& O. Vásquez. 2015. Ponthieva luegii (Orchidaceae), a new taxon from the Karst formations of Guatemala. Richardiana 15: 224-231. 
Bertolini, V., A. Damon, F.R. Luna Tavera \& A.N. Rojas Velázquez. 2012. Las orquídeas del Valle del Mezquital, Hidalgo (México), resultados preliminares. Revista Chapingo Serie Zonas Áridas 11(2): 85-94.

DRESSLER, R. L. 1990. The orchids natural history and classification. Harvard University Press, Cambridge. 332 pp.

INEGI (Instituto Nacional de Estadística y Geografía, México). 2008. Anuario estadístico Hidalgo 2008, Instituto Nacional de Estadística y Geografía, Gobierno del Estado de Hidalgo. Pachuca. 103 pp.

Olson, D.M., E. Dinerstein, E.D. Wikramanayake, N.D. Burgess, G.V.N. Powell, E.C. Underwood, J.A.D. Amico, I. ItouA, H.E. Strand, J.C. Morrison, J. Loucks, T.F. Allnutt, T.H. Ricketts, Y. Kura, J.F. Lamoreux, W. Wesley, P. Hedao \& K.R. Kassem. 2001. Terrestrial Ecoregions of the World : A New Map of Life on Earth. Bioscience 51: 933-938.

Pérez-Escandón, B.E., M.A. Villavicencio-Nieto \& A. RamírezAguirre. 2003. Lista de las plantas útiles del estado de Hidalgo. Universidad Autónoma del Estado de Hidalgo Centro de Investigaciones Biológicas, Pachuca. 136 pp.

Rzedowski, J. 2006. Vegetación de México. 1ra. Edición Digital, Comisión Nacional para el Conocimiento y Uso de la Biodiversidad, Ciudad de México. 504 pp.

Schultz, J. 2002. The Ecozones of the World. The Ecological
Divisions of the Geosphere. Springer, New York. 252 pp.

Semarnat (Secretaría de Medio Ambiente y Recursos Naturales). 2010. Norma Oficial Mexicana NOM-059SEMARNAT-2010, Protección ambiental, Especies nativas de México de flora y fauna silvestres, Categorías de riesgo y especificaciones para su inclusión, exclusión o cambio, Lista de especies en riesgo. Diario Oficial de la Federación, 30 de diciembre de 2002.

Soto-Arenas, M.A. 1996. Mexico (Regional account). In: IUCN/ SSC Orchid Specialist Group. 1996. Orchids - Status Survey and Conservation Action Plan, pp. 53-58. IUCN, Gland Switzerland and Cambridge, UK.

Soto-Arenas, M.A., E. Hágsater, R. Jiménez Machorro, G.A. Salazar Chávez, R. Solano Gómez, R. Flores Gonzáles \& I. Ruiz Contreras. 2007. Las Orquídeas de México. Catálogo Digital. Disco interactivo multimedia Win-Mac. Herbario AMO, Insituto Chinoin, A.C. México. 304 pp.

SzlachetKo, D. \& P. RutKowski. 2000. Gynostemia Orchidalium I. Acta Botanica Fennica 169: 288-296.

WCSP (World Checklist of Selected Plant Families). 2015. Facilitated by the Royal Botanic Gardens, Kew. Published on the Internet: http://apps.kew.org/wcsp/Retrieved $12 / 02 / 2015$.

Recibido: 29.10.15

Aceptado: 05.09.16 